\title{
Aplicación del análisis de regresión para el estudio de las pymes productivas españolas que obtienen informes motivados vinculantes de proyectos de $\mathrm{I}+\mathrm{D}+\mathrm{i}$
}

\section{Application of the regression analysis for studying Spanish producers SMEs get binding motivated reports about projects $R \& D \& 1$}

\section{口nu}

Ana-Isabel Pérez-Molina', Victor Gisbert-Soler² y Pablo Diaz-Garcia ${ }^{3}$

Universitat Politècnica de Valencia (España)

DOI: http://dx.doi.org/10.6036/8249

La innovación en las Pymes en base a proyectos de $I+D+i$ es un factor clave para el desarrollo empresarial. Coexisten estudios cuantitativos relacionados con la $\mathrm{I}+\mathrm{D}+\mathrm{i}$, pero si una sociedad pretende innovar no dispone de información sobre las estructuras, formas de trabajar, etc. que utilizan las entidades que ya innovan [1].

Para modelizar en base el análisis de regresión a las pymes productivas españolas que realizan proyectos de $I+D+i$ se ha analizado una muestra de 80 empresas españolas con una metodología de análisis propia [2], se ha caracterizado dicha muestra con un análisis univariante y bivariante [3], se ha aplicado un análisis discriminante [4], y finalmente se ha realizado un análisis de regresión [5]. La característica común de dichas compañias es que han conseguido al menos un Informe Motivado Vinculante, en alguna de las anualidades trabajadas.

Se han analizado diversas variables y se han obtenido modelos de regresión que permiten obtener una ecuación que determina el valor de una variable dependiente en función de diversas variables independientes. Concretamente se han trabajado las siguientes variables [13]:

Número de trabajadores. Existen variables comunes a diversos modelos: Número de trabajadores de los años anteriores; Número de personas que se dedican a los proyectos (en sus diferentes tipologías); Existencia de plan estratégico general y de I+D+i o tecnológico; Nivel de estructuración del departamento de $\mathrm{I}+\mathrm{D}+\mathrm{i}$; Valoración del grado de evidencias de los proyectos; Participación del gerente en los proyectos y gasto aceptado por el MINECO en la partida personal interno en
I+D+i en los proyectos. Estas regresiones han permitido verificar que el tamaño de las empresas no es un factor determinante en la mayoría de las características cualitativas de los proyectos de I+D+i.

Facturación / número de empleados. Las variables más influyentes son: Facturación de los años anteriores; Número trabajadores en la anualidad 2006; Número de personas dedicadas a la $\mathrm{I}+\mathrm{D}+\mathrm{i}$ (diferentes tipologías); Gastos aceptados por el MINECO en las diferentes partidas de los proyectos; Problemas para financiar la $\mathrm{I}+\mathrm{D}+\mathrm{i}$ y mecanismos de comunicación interna. Estas regresiones no han permitido validar la hipótesis de que las empresas con mayor gasto en $\mathrm{I}+\mathrm{D}+\mathrm{i}$, facturan más por persona ocupada.

Gasto justificado en I+D+l. Las variables que más influyen en el gasto justificado en I+D+i son: Número de personas dedicadas a la $\mathrm{I}+\mathrm{D}+\mathrm{i}$ (en sus diferentes tipologías), en la anualidad analizada y en las anteriores; Nivel de aprovechamiento de las ayudas públicas; Inversiones elementos inmovilizado material e intangible y gastos aceptados por el MINECO en las diferentes partidas, en la anualidad analizada y en las anteriores.

Presupuesto del proyecto. Se dispone de varias rectas de regresión capaces modelar el presupuesto total $(1+D+i)$ del proyecto y el presupuesto en $I+D$, pero no se ha conseguido ningún modelo que represente el presupuesto en IT. Los parámetros influyentes son: Número de personas dedicadas al proyecto (en sus diferentes tipologías); Duración del proyecto; Las cantidades subvencionadas a fondo perdido, los créditos preferentes y las cantidades prestadas por entidades financieras.

Gasto justificado del proyecto. Se han conseguido rectas de regresión que modelan el gasto justificado total $(1+D+i)$ y el gasto justificado en I+D de los proyectos, ahora bien, no se ha conseguido ningún modelo para el gasto en IT. Los parámetros más influyentes son los mismos que afectan al presupuesto de los proyectos.

Gasto aceptado por el MINECO del proyecto. Se han conseguido rectas de regresión que modelan el gasto aceptado total $(I+D+i)$, el aceptado en $I+D$ y el aceptado en IT. Las variables más influyentes son: Número de personas dedicadas al proyecto (en sus diferentes tipologías); Aprovechamiento de las ayudas públicas; Cantidades prestadas por entidades financieras; Gastos justificados y el hecho de que la novedad tecnológica sea nacional, internacional o interna.

En resumen, se han conseguido relaciones complejas entre los diversos aspectos de las pymes productivas españolas que realizan proyectos de I+D+i, consiguiéndose por lo tanto, modelos basados en rectas de regresión capaces de asistir a las empresas españolas hacia conseguir incluir la $++D+i$ en su estrategia empresarial.

\section{REFERENCIAS}

[1] Ministerio de Economía y Competitividad. "Informes Motivados para deducciones fiscales por actividades de I+D e innovación tecnológica". 2012.

[2] Pérez-Molina Al, Díaz-García P, GisbertSoler, V. "Development of a systematic analysis for the characterization of spanish productive SME performing projects REDEt|". International scientific Conference. Innovative solutions for sustainable development of textiles and leather industry. May, 23rd - 24 th, 2014 Oradea/Romania. University of Oradea, 2014. Issn: 1843-813X.

[3] Pérez-Molina Al, Gisbert-Soler, V, Díaz-Garcia P. Caracterización de pymes productivas españolas que realizan proyectos de I+D+i en base a Informes Motivados Vinculantes. DYNA Management. DOI: http://dx.doi.org/10.6036/ MN7840

[4] Pérez-Molina Al, Díaz-García P, Gisbert-Soler, V. Análisis discriminante aplicado a la pymes productivas españolas que realizan proyectos de I+D+i. DYNA Management. http://dx.doi. org/10.6036/MN7925

[5] Pérez-Molina Al, Gisbert-Soler V, DiazGarcía P. Aproximación inicial al análisis de regresión aplicado a la pymes productivas españolas que realizan proyectos de I+D+i. DYNA Management. http://dx.doi. org/10.6036/MN7926 


\section{2-6 OCTUBRE 2017}

RECINTO GRAN VIA

\section{CHEMISTRY FOR THE FUTURE}

- Sea el primero en llegar al futuro participando

como experto, expositor o visitante.

- Inscríbase en nuestras actividades de

networking, jornadas técnicas y congresos.

- No se lo pierda, solo ocurre una vez cada tres años. Entre en www.expoquimia.com

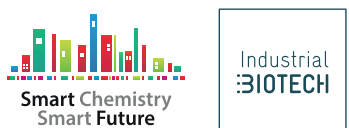

\begin{tabular}{c|c}
$\begin{array}{c}\text { Industrial } \\
\text { Smart Chemistry } \\
\text { Smart Future }\end{array}$ & :310TECH
\end{tabular}

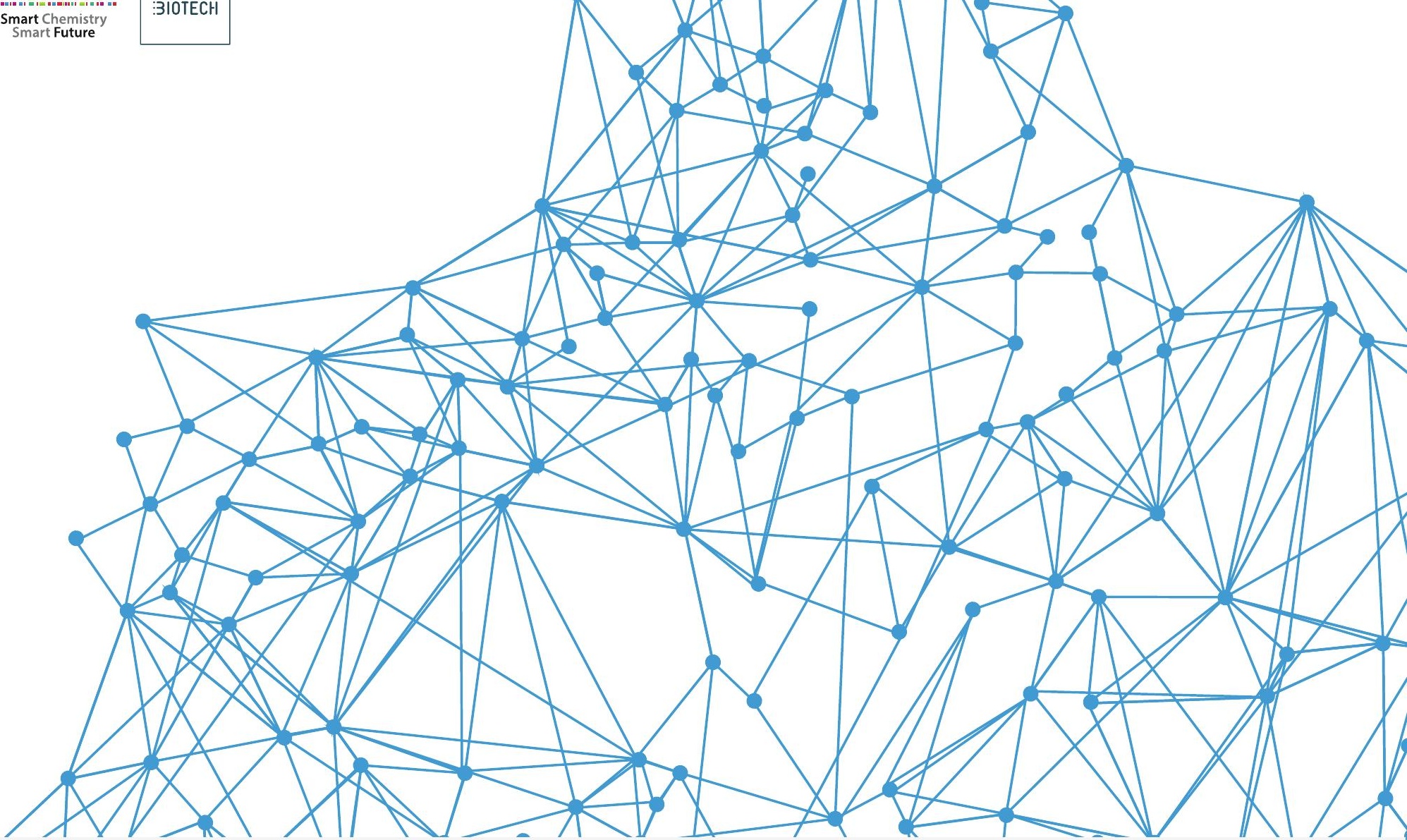

Co-located events: $\begin{aligned} & \text { WORLD } \\ & \text { CHEMICAL } \\ & \text { SUMMIT }\end{aligned}$ 